\title{
Implementation of an Automated System for Optimal Pump Control ${ }^{+}$
}

\author{
Matthew Main ${ }^{1, *}$, Marcus Fowler ${ }^{1}$ and Drummond Modley ${ }^{2}$ \\ 1 Servelec Technologies, Spring Court, Station Road, Dorking RH4 1EB, UK; \\ marcus.fowler@servelec-technologies.com \\ 2 Wessex Water, Claverton Down, Bath BA2 7WW, UK; Drummond.Modley@wessexwater.co.uk \\ * Correspondence: matthew.main@servelec-technologies.com; Tel.: +44-1306-742-772 \\ + Presented at the 3rd EWaS International Conference on "Insights on the Water-Energy-Food Nexus", \\ Lefkada Island, Greece, 27-30 June 2018.
}

Published: 1 August 2018

\begin{abstract}
To support their Integrated Water Supply Grid, Wessex Water recognized the need for a sophisticated control system. The Servelec Technologies pump optimization system OptiMISER was identified as the best tool for this function and has been in use in the Control Room since September 2014. Since then it has been controlling pumps and valves across the Warminster area, ensuring pro-active optimal management of that part of the network, as a precursor to full implementation over the Grid.
\end{abstract}

Keywords: energy management; optimization; pump scheduling; automated network control

\section{Introduction}

Over the last 5 years, Wessex Water have undertaken a $£ 230$ million project improving connections between existing infrastructure in order to provide a grid which fully integrates their water supply network, allowing them to meet water demand now and in the future, improve resilience and security of supply to end users, whilst managing reduced abstraction consents and deteriorating raw water quality.

To support their Integrated Water Supply Grid, Wessex Water [1] recognized the need for a sophisticated control system. A key part of the evaluation of potential suppliers was a fully automated pilot undertaken in the spring of 2012. This tested the ability of systems to provide pump schedules reliably, which were both cost-optimal and operating within network constraints, under both ordinary and strained (i.e., outage) conditions. The Servelec Technologies pump optimization system OptiMISER was identified as the best tool for this function and has been in use in the Control Room since September 2014.

Since then it has been controlling pumps and valves across the Warminster area, ensuring pro-active optimal management of that part of the network, as a precursor to full implementation over the Grid.

The system has been expanded by Wessex Water since the original Phase 1 implementation. As of early 2018, 22 pump sets and 2 flow control valves are routinely being controlled. Wessex Water themselves are continuing to extend the area under control to incorporate their new Integrated Water Supply Grid and have plans to roll out control to their entire supply area, spanning some 180 sources and treatment works, containing in the region of 600 pump sets. 


\section{Process}

\subsection{System Overview}

The optimization approach employed to pump scheduling is based on a model of the water supply network. The decision variables are pump statuses and valve positions and the flows, volumes and costs resulting from these. The optimization is framed in half-hour blocks of pumping to coincide with energy metering tariff charging. The software can also handle 15-min timesteps, e.g., for immediate outage response.

The model incorporates the wide range of network constraints relevant to the system operation, including licenses, pump performance, flow capacities, water quality, pressures, storage and demand. The same model is used in Wessex Water for both short-term operational planning and longer-term production planning and water resources analysis.

The optimization horizon for pump scheduling is user-defined and can vary from a few hours to several weeks. Wessex Water regularly utilize a 72-h optimization producing a schedule over $48 \mathrm{~h}$. This ensures the optimization takes into account planned outages, demand variations, license usage and varying tariff rates over the horizon. 7 a.m. refill targets are employed and source abstraction targets are implemented from longer-term plans. Regular optimization makes best use of prevailing conditions, to react to changes in storage network outages and any overrides implemented by users.

The primary output from OptiMISER is a set of control instructions which are automatically exported to the central SCADA system for implementation. A schedule of actions is also produced for Operations Centre staff to monitor activity against planned operation throughout the day, in the form of pump switches and valve positions over time. In addition, trajectories of storage, demand, abstraction and key flows around the supply system are produced in order to assist in real-time performance monitoring (as shown in Figure 1).

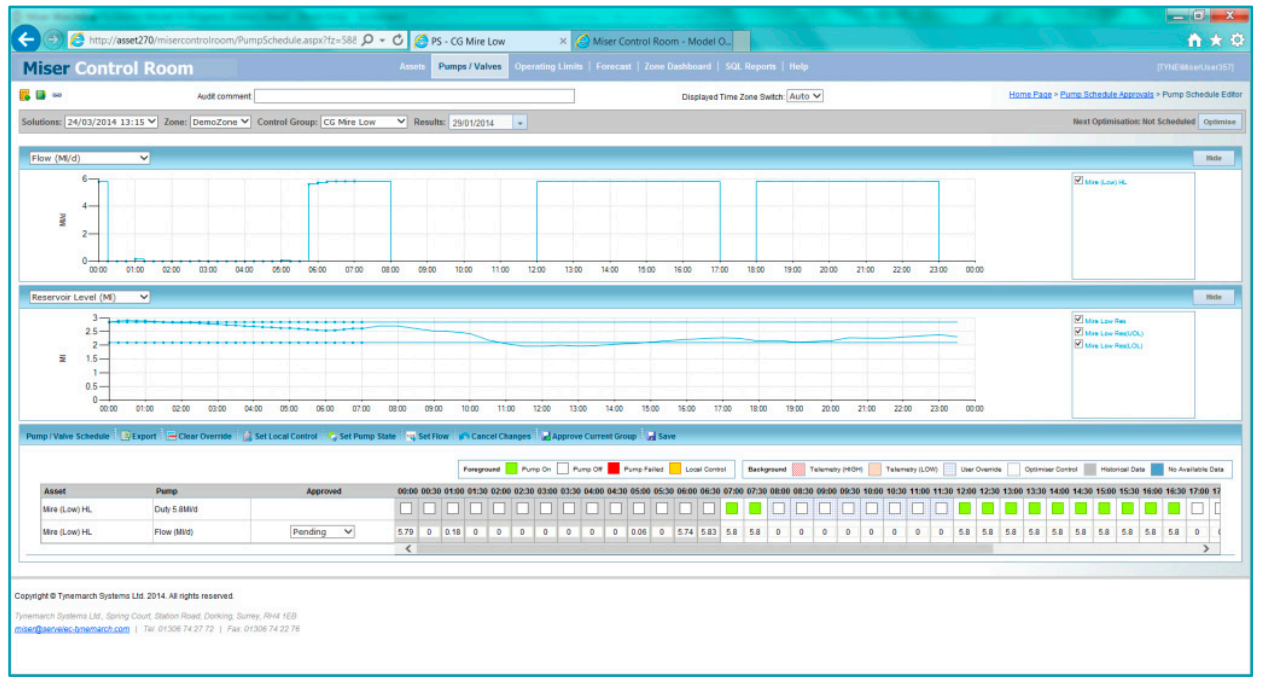

Figure 1. OptiMISER Schedule Editor Interface.

It is imperative that the whole process can be completed within a 30-min window. In practice, the process as a whole routinely takes just a few minutes, with data from telemetry imported and processed to produce reservoir levels and demands etc. within a minute, optimizations taking about 1-2 min to produce the latest instructions for export, and data export to SCADA then also finishing within a minute.

\subsection{Model Configuration}

Underlying the system is a model of the relevant area of the network. Wessex Water have been long-term users of the Servelec Technologies modelling and optimization system MISER for 20 years, 
since the late 1990s. They therefore held a compatible existing model. The model schematic for part of the Phase 1 area in Wessex Water is shown in Figure 2 below.

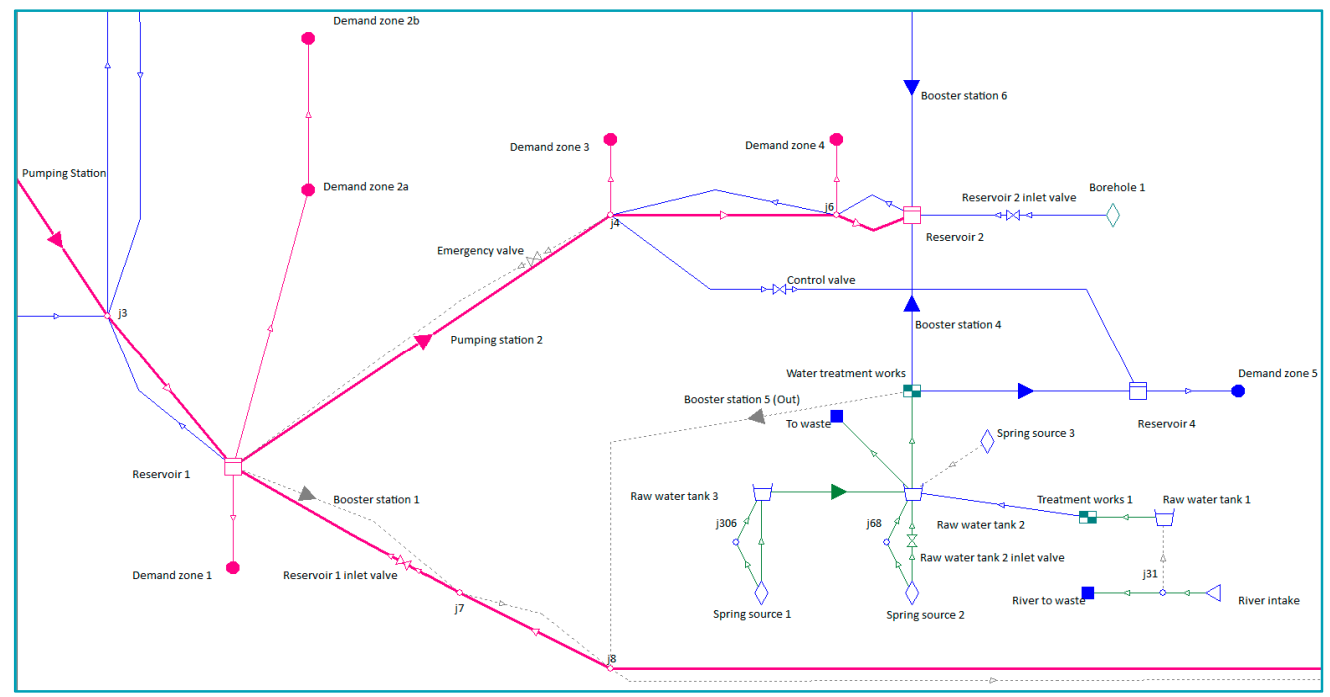

Figure 2. OptiMISER Phase 1 Area Schematic (part; anonymised).

The model was constructed using the OptiMISER client interface, with configuration data including:

- Abstraction limits from licenses, monthly plans, river flows and spring yields

- Costs for chemicals and sludge treatment, and electricity tariffs

- Pump performance data and operating rules for pump switching and rotation, as well as pump inhibit levels

- Maximum/minimum flows/pressures, maximum rates of flow change

- Sweetening and conditioning flow requirements, where regular flows must be maintained along pipelines, either as high flow pulses, for instance to meet PODDS conditioning requirements, or as minimum sustained flow rates and throughput over time

- Service reservoir capacities, operating limits and target levels

As described by Bene [2], a diverse range of optimization techniques can be employed for pump scheduling applications, and no universal preference exists as to which theory is most practicable. The techniques used are broadly divided into two main schools:

- iterative hydraulic modelling, typically accompanied by genetic algorithms, and

- $\quad$ Mixed Integer Linear Programming

As the strategic supply system is considered, the need for explicit modelling of all pressures across the network is reduced. A MILP approach to optimization is therefore favored ahead of Genetic Algorithms. There are variable speed pumps within the network, and one area with significant levels of interaction in pressure, with changes in customer demand altering pump output. These are incorporated with sufficient accuracy using piecewise linear curves. The MILP approach allows for such constraints only to be included where necessary, reducing the computational overhead required.

\subsection{Telemetry Data Connection}

Digital and analogue signals are imported every 15 min to provide the current network state, including recent flows and pressures and prevailing reservoir levels, pump running/stopped status and availability, and digital alarms. The context of OptiMISER interacting with users and other systems is shown in Figure 3. 


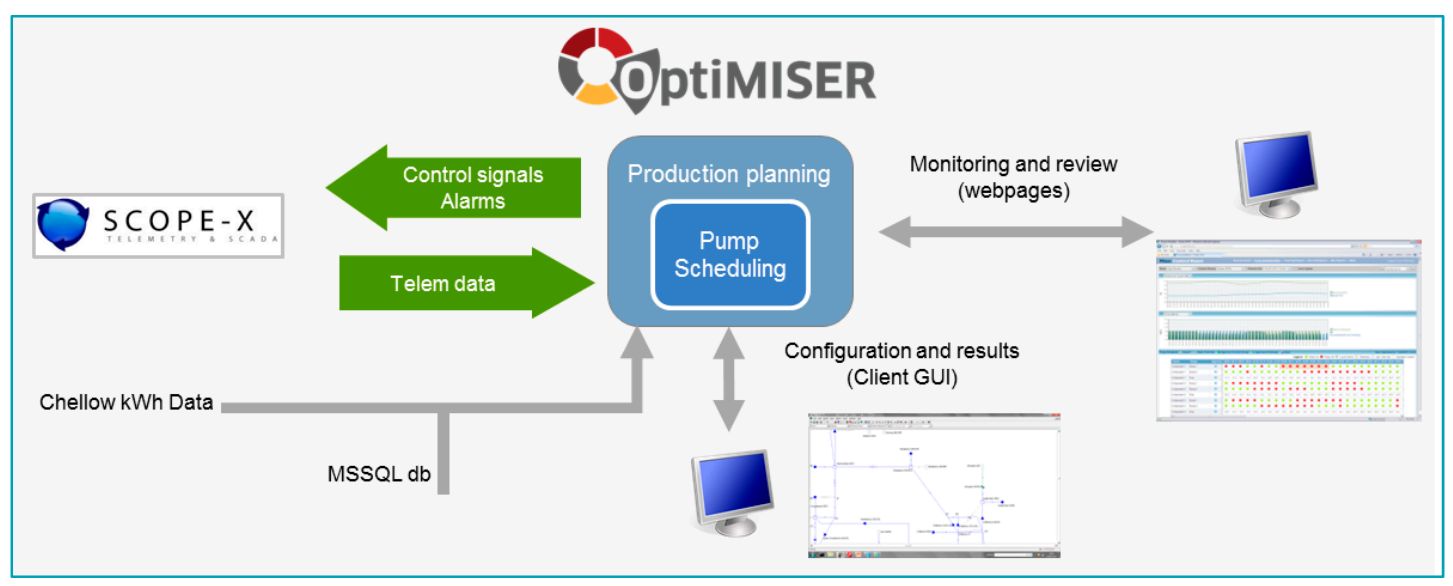

Figure 3. OptiMISER Context Diagram.

Analogue data are validated against a range of parameters before use and replaced if necessary. Where alternative meters or telemetry points are available, these are automatically used on failure of the primary point. Where no alternative meter is available, data can be replaced using a statistical infilling algorithm based on valid historical data, transformed to meet the values either side of the invalid period.

Analogue data are processed to produce historical and reservoir volumes, flows (e.g., to monitor daily license usage) and demand values.

Reports are automatically produced on the validation of telemetry data, including both the raw values imported and any replacements made, and which validation algorithms have been failed. In addition to the automatic data cleansing, corrections can be made manually either to identify periods of meter error or to provide replacement values.

Telemetry alarms, such as in response to poor water quality, TRIAD warnings and pump outages, are imported via digital signals and used directly by OptiMISER to modify constraints within the model. This includes:

- $\quad$ automatically preventing assets from being scheduled for use when they are unavailable

- modelling where valves freeze as a result of power cuts, i.e., preventing the optimizer from specifying a change in flow rate.

- a turbidity alarm which prevents an increase in flow rate. This site is also limited in how much it can increase its flow rate above its highest flow rate over the preceding $24-\mathrm{h}$ period.

The same telemetry connection is also used to export control instructions from OptiMISER to the SCADA system for immediate implementation. Instructions can be issued in the form either of analogue signals, such as flow rates, or as digitals to control pump and valve status. Pump control differs from site to site in being applied at either the individual pump level or higher pump-set level.

\subsection{Demand Forecasting}

The latest analogue telemetry data are used to forecast demand from the recent series at 15-min intervals over the coming $72-\mathrm{h}$ period. Many individual forecasts are produced, typically one per service reservoir or storage tank.

Demand series are split between the sequence of daily average values and normalized diurnal patterns. Auto-regressive models are used to forecast the average daily demand at each node.

Exponential smoothing algorithms are approximated in forecasting the diurnal aspect of demand from the same days of previous weeks.

This approach allows a good forecast to be produced for a range of patterns of demand, providing a particularly high standard of forecast for domestic demands. This also means that manual changes to the system, such as rezoning or changes to bulk transfers, are accounted for automatically. So-called Event Days can be specified on which adjustments are made to the forecasts, e.g., to reflect increases resulting from local festivals. 
These combined models typically generate forecasts which have coefficients of determination of $90 \%$ or above, with the auto-regressive daily sequence producing coefficients of determination typically of $75-80 \%$. An example of recent forecast compared with actual data from a two-week period in December 2017 is shown in Figure 4 above.

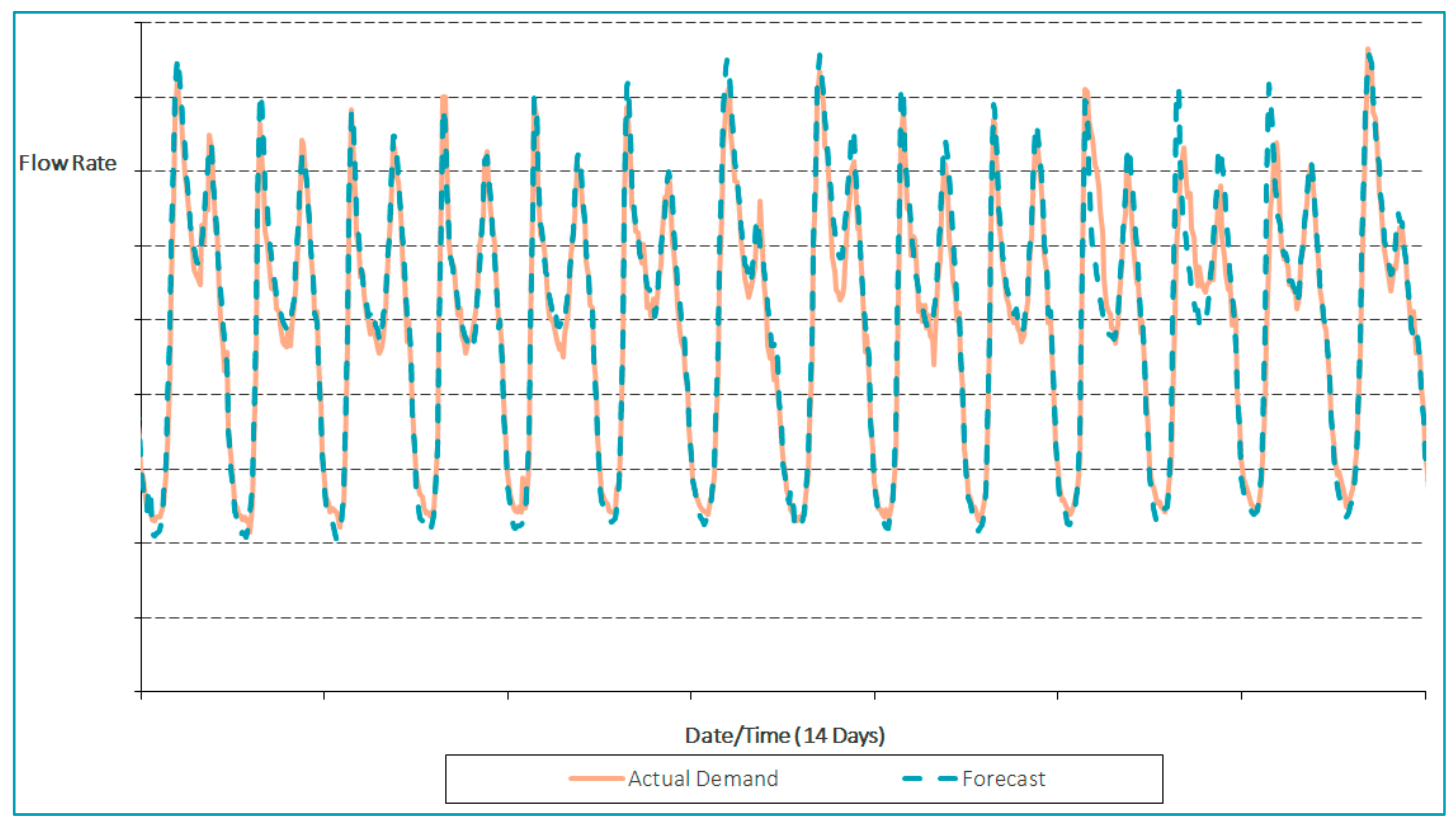

Figure 4. Demand Forecasting Model Fit.

\subsection{Optimization}

Every $30 \mathrm{~min}$, an optimization of the network is carried out. Flows, pump and valve statuses, reservoir volumes, and network pressures are considered as variables in the optimization problem.

The objective within pump scheduling is to minimize energy and treatment costs subject to meeting a range of operational constraints and targets, including expected demands, constraints on storage, license conditions, plant availability, and allowable pump combinations.

The problem is formulated in 30-min timesteps with pump statuses, flows and pressures assumed to be static within each timestep. Non-linear constraints such as pump and valve statuses, and if/then control rules, are included. The problem is formulated as a Mixed-Integer Linear Programming problem and solved with a commercially available MILP solver (FICO Xpress). This is carried out over the defined 72-h horizon taking account of the forecast demands and planned outages.

In order to reliably complete the optimization in a short space of time, providing pump instructions within a couple of minutes, the problem is formulated as a combination of fully-modelled 30-min timesteps in the short-term and approximated (e.g., linearized and longer) timesteps to the horizon end, which are gradually overwritten by the fully-modelled timesteps as the optimization proceeds.

The optimization operates within a set of inflexible constraints and flexible targets or goals. Some objectives which might ordinarily be considered to be absolute constraints, such as meeting demand, are included as high priority but flexible goals. The problem is formulated as a sequence of mixed integer optimizations, each with a different objective function. The result of one objective function is carried forward to the subsequent pass as a fixed constraint. This approach allows a feasible solution to be found for almost all input parameters and provides forewarning of network problems, e.g., storage unavoidably emptying and risks to supply.

OptiMISER must produce a pump schedule which seeks to manage service reservoir and other tank storage such that user-specified targets are met at key points throughout the optimization period. For example, targets are applied to refill service reservoirs at 07:00 each morning to maximize storage for morning demands. Flexible daily abstraction limits are also used to represent 
longer-term constraints on output, such as from planned use of annual abstraction licenses or resource availability.

Additional objectives are more subtle but also must be specified. An objective is included to minimize the number of pump switches in order to produce schedules which are realistic in terms of practical implementation. Furthermore, constraints are specified which limit the number of switches in any given time period, although constraints of this nature must be configured in such a way as to become flexible when an outage or other emergency situation takes priority.

This leads to a complex list of competing objectives. as above, multiple optimization goals are considered in a prioritized sequence. Meeting demand is typically considered at highest priority. Although minimizing cost is a key aim of OptiMISER, this is configured at lower priority than respecting reservoir targets and operating limits. The sequence of priorities is fully configurable and the lowest priority goals, including minimizing pump and valve switches, are weighted against cost. This allows the OptiMISER to provide a solution which is both practicable to implement while delivering cost efficiencies.

\subsection{Control Room}

Output is in the form of flows, pump statuses, and reservoir levels planned at half-hourly steps over the coming 48-h period. The optimization results, as well as the current and recent state of the network, are monitored via a set of web pages.

These web pages are interactive, allowing Control Room staff to make operational overrides. For example, using the Schedule Editor screen, as shown in Figure 1, operators can:

- $\quad$ Review and approve schedules

- Specify overrides to current and future operation, including:

- pump outages (for planned outages)

- pump must-runs (e.g., for water sampling)

- pump and valve flow rates to specify operation

- target reservoir levels, e.g., to refill res to $90 \%$ at 7 a.m., and to reduce to $60 \%$ at 5 p.m.

- As an optimization takes place every $30 \mathrm{~min}$, a revised schedule will be produced automatically to meet these constraints optimally according to the appropriate priorities.

- Receive alarm signals and early warning of any resultant difficulties in the network, such as low reservoir levels from network outages

- Receive reports on the state of the network and the standard of optimization, including where any high priority optimization objectives have not been met. These reports are customized by individuals so that information appropriate to a range of applications, from operations to IT and management, can automatically be generated from the same source.

\section{Outcomes}

Wessex Water have identified a range of benefits underpinning the success of the system. By allowing an holistic view of the supply network over the days ahead to be taken, OptiMISER has introduced profoundly different ways of controlling the water supply sources and network.

A typical 48-h pumped output for one pumping station in the region controlled is shown in Figure 5 . The period of highest electricity tariff, late afternoon, is clearly avoided, where this was not routinely the case under the previous control regime. An increase in overnight pumping, when electricity is cheapest, is also observed. 


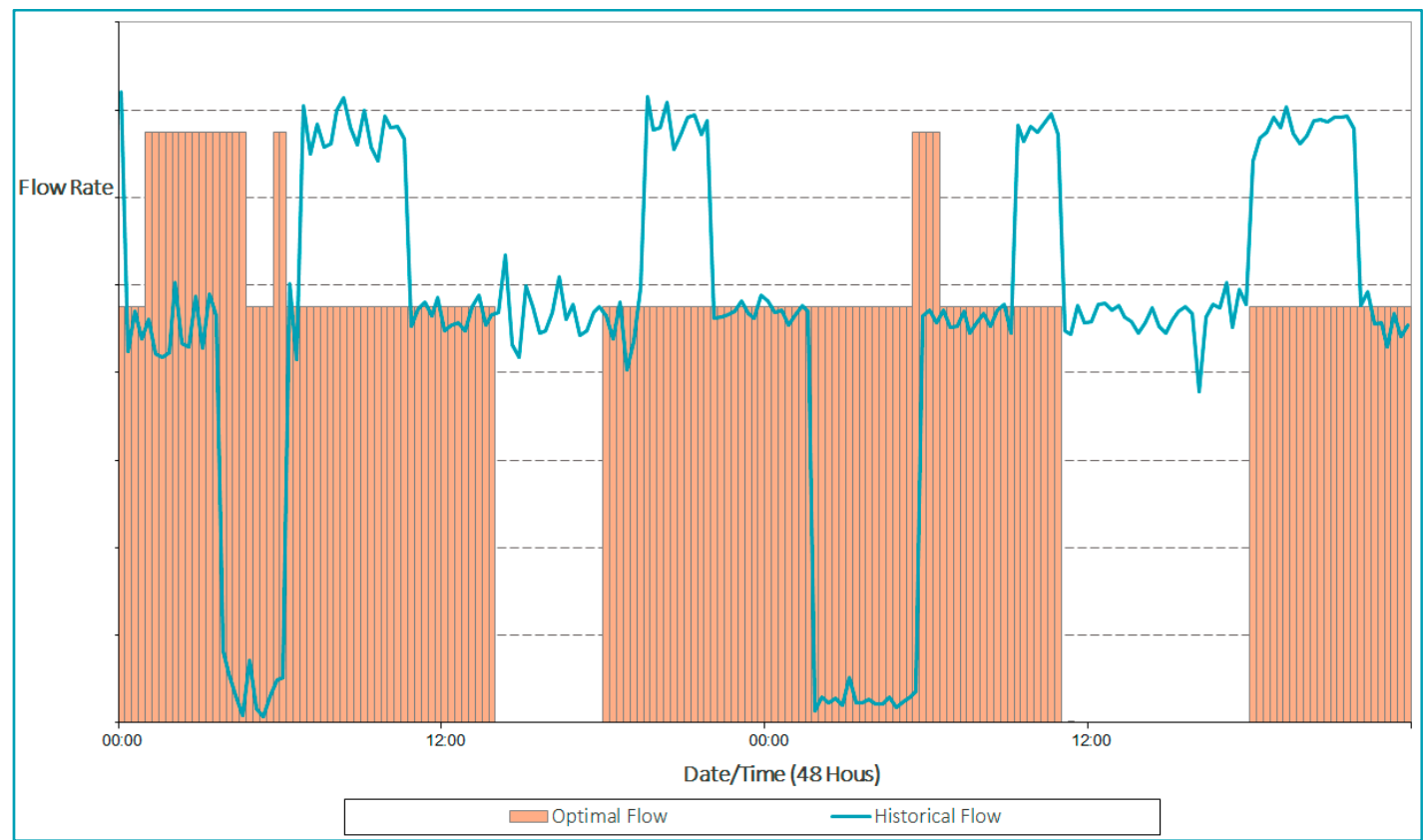

Figure 5. Sample Flow Results.

Wessex Water have been able to report a reduction of OPEX on site of $10-15 \%$, in $£ / M L$ terms. Furthermore, they have assessed the success of the system as follows:

"The optimiser has introduced a radically different way of controlling water supply sources and networks.

It allows an holistic view to be taken over the supply network and over the days ahead-rather than decisions being based on only the current water level in the adjacent reservoir. This gives many benefits, including:

- Improved security of supply and contingency planning

- Efficient transfer of flows in a complex supply system

- Efficient management of assets in a complex transfer and distribution system

- $\quad$ Savings made in avoiding TRIADs and DUoS periods

- Optimal outage planning

- Optimal blending ratios for nitrate sources

- Reduced call outs out-of-hours as forecast provides more confidence in networks flexibility"

\section{Conclusions}

A range of benefits have been identified by Wessex Water and the practicality of on-line, closed-loop, optimal control of pumps has been clearly demonstrated. Wessex Water have gained confidence in the system and are rolling it out to key parts of their network.

\section{Glossary of Abbreviations}
Abbreviation
Definition
DUoS
Distribution Use of System Charges. Used to refer to period of high electricity tariff in UK energy supply.
MILP Mixed Integer Linear Programming
OPEX Operational Expenditure
PODDS Prevention of Discoloration in Distribution Systems. Periods of high flow rate are specified to clean the inside of distribution mains.
SCADA Supervisory Control and Data Acquisition. System transmitting telemetry data and control signals.
TRIAD Period used to calculate the Transmission Network Use of System charges in UK energy supply. The three peak periods of energy use in each winter, used to retrospectively determine charges. 


\section{References}

1. Modley, D.; Wood, M.; Muscat, C.; Lowe, L.; Bilton, S.; Fowler, M.R. Water Projects Online 2016. Water Supply GRID_Optimiser System. Available online: http://www.waterprojectsonline.com/case_studies/ 2016/Wessex_GRID_Optimiser_2016.pdf (accessed on 21 May 2018).

2. Bene, J.G. Pump Schedule Optimisation Techniques for Water Distribution Systems. Ph.D. Thesis, University of Oulu, Oulu, Finland, 2013.

3. Alrheeh, M.; Mahmoud, H. Using Genetic Algorithms in Pump Scheduling to Reduce the Pumping Cost. Damasc. Univ. J. 2009, 25, 95-106. Available online: http://www.damascus-university.edu.sy/mag/eng/ images/stories/erhbeh.pdf (accessed on 21 May 2018).

4. Pasha, M.F.K.; Lansey, K. Optimal Pump Scheduling by Linear Programming, World Environmental and Water Resources Congress, 2009. Available online: https://ascelibrary.org/doi/pdf/10.1061/41036\%28342\% 2938 (accessed on 21 May 2018).

5. Menke, R.; Abraham, E.; Parpas, P.; Stoinav, I. Exploring Optimal Pump Scheduling in Water Distribution Networks with Branch and Bound Methods. Water Resour. Manag. 2016, 30, 5333-5349. Available online: https://link.springer.com/article/10.1007/s11269-016-1490-8 (accessed on 21 May 2018).

6. Husband, S.; Boxall, J. Predictive water quality modelling and resilience flow conditioning to manage discolouration risk in operational trunk mains. J. Water Supply 2015, 64, 529-542.

7. National Grid, Introduction to Triads, 2015. Available online: https://www.nationalgrid.com/sites/ default/files/documents/44940-Triads\%20Information.pdf (accessed on 21 May 2018).

(C) 2018 by the authors. Licensee MDPI, Basel, Switzerland. This article is an open access article distributed under the terms and conditions of the Creative Commons Attribution (CC BY) license (http://creativecommons.org/licenses/by/4.0/). 